14[L].-James Nicholas Sarmousakis (1912- ), Tables of associated Legendre functions with complex argument. Manuscript and film in possession of the author. Film copies at the Mathematical Tables Project, New York City, and in the Library of Brown University.

The Tables of $P_{n}{ }^{m}(i x)$ and of $Q_{n}{ }^{m}(i x)$ are as follows:

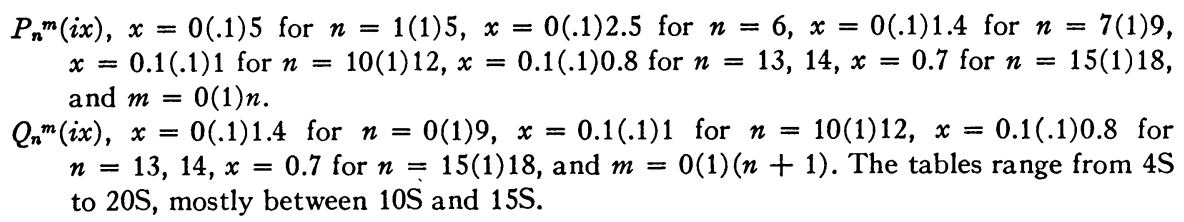

The computation of these tables was done only once and the sole auxiliary tables used in their preparation were the Tables of the First Ten Powers of the Integers from 1 to 1,000, New York, Project for the Computation of Mathematical Tables, 1938. A few miscellaneous checks have been made. As yet there has been no appropriate rounding off to a suitable number of significant figures.

I have used the tables to evaluate relative dissociation constants of substituted benzoic acids which were computed on the basis of an electrostatic theory of substituent effects.

Aberdeen Proving Grounds

J. N. SARmousakis

Such tables are also of use for computing potentials, flow and diffusion about disks and rods.-EDIToR.

\title{
MECHANICAL AIDS TO COMPUTATION
}

6[Z].-Simpson Leroy Brown (1881- ), “A mechanical harmonic
synthesizer-analyzer," Franklin Institute, Jn., v. 228, 1939, p. 675-694.

The 30-term synthesizer-analyzer described in this paper is perhaps the largest machine of its kind having so few harmonic components. It is 15 feet long and 7 feet high and weighs nearly a ton. This may be compared with the old Michelson and Stratton ${ }^{1} 80$-term machine which is about 3 feet long and 4 feet high. The reasons for building the machine on a large scale were to gain accuracy and to save labor. The driving mechanism, for example, is a train of 22 spur gears which, though of only commercial grade, are large enough so that more accuracy is obtained than with small expensive precision made gears.

Two simple harmonic motions, out of phase by $90^{\circ}$, are generated at both ends of 15 rotating shafts by Scotch crossheads. This gives 15 sine terms and 15 cosine terms in the corresponding Fourier series. The two fundamental motions are capable of amplitudes up to 16 centimeters while even the components of highest frequency may be set with amplitudes as great as 4 centimeters. Thus the machine draws curves well over a foot in overall width. The 30 harmonic motions are added, in the usual manner, by an endless fine chain passing over pulleys.

Harmonic analysis to, say, 60 terms could be performed by pencil and paper methods, better still by commercial computing machines; it may also be done entirely mechanically with a 60 -term analyzer. The author points out that a combination of the two methods is not only possible but indeed desirable, and that by using an accurate synthesizer with comparatively few harmonic elements one can make analyses that include many harmonic elements by applying a little pencil and paper before and after using the analyzer.

This may be illustrated as follows. Let $y=f(x)$ be the subject of analysis, a periodic function defined empirically by 64 of its equally spaced arguments $\left(y_{1}, y_{2}, \cdots y_{64}\right)$. We wish 
to find constants $A_{n}$ and $B_{n}$ so that

$$
y=f(x)=\sum_{n=1}^{31}\left(A_{n} \cos \frac{2 \pi n x}{64}+B_{n} \sin \frac{2 \pi n x}{64}\right)
$$

Our machine will calculate expressions of the type

$$
Y=\sum_{m=1}^{15}\left(a_{m} \cos \frac{2 \pi m x}{32}+b_{m} \sin \frac{2 \pi m x}{32}\right)
$$

Before using the machine we prepare 60 constants

$$
a_{m}, \quad b_{m}, \quad a_{m}{ }^{\prime}, \quad b_{m}{ }^{\prime}, \quad(m=1,2, \cdots, 15)
$$

defined in terms of the given $y$ 's by

$$
\begin{aligned}
a_{m} & =y_{m}-y_{32-m}-y_{32+m}+y_{64-m} \\
b_{m} & =y_{m}+y_{32-m}-y_{32+m}-y_{64-m} \\
a_{m}^{\prime} & =y_{m}+y_{32-m}+y_{32+m}+y_{64-m} \\
b_{m}^{\prime} & =y_{m}-y_{32-m}+y_{32+m}-y_{64-m}
\end{aligned}
$$

Setting the amplitudes $a_{m}, b_{m}$ on the machine we draw the curve represented by (1) and take therefrom 64 equally spaced ordinates $\left(Y_{1}, Y_{2}, \cdots, Y_{64}\right)$. Actually, no curve need be drawn and measured; the machine simply is stopped and read 64 times at equal angle intervals. Similarly $a_{m}{ }^{\prime}$ and $b_{m}{ }^{\prime}$ lead to $\left(Y_{1}{ }^{\prime}, Y_{2}{ }^{\prime}, \cdots, Y_{64}{ }^{\prime}\right)$. The desired numbers $A_{n}$ and $B_{n}$ may now be found as simple combinations of the $Y, Y^{\prime}$, and $y_{18}, y_{32}, y_{48}, y_{64}$. Thus for $j=1,2, \cdots, 16$ we have

$$
\begin{aligned}
64 A_{2 j-1} & =Y_{2 j-1}+Y_{64-2 i+1}+2\left(y_{64}-y_{32}\right) \\
64 B_{2 j-1} & =Y_{2 j-1}-Y_{64-2 i+1}+2(-1)^{i}\left(y_{48}-y_{16}\right) \\
64 A_{2 j} & =Y^{\prime}{ }_{2 j}+Y^{\prime}{ }_{64-2 j}+2\left(y_{64}+y_{32}\right)+2(-1)^{i}\left(y_{48}+y_{16}\right) \\
64 B_{2 j} & =Y^{\prime}{ }_{2 j}-Y^{\prime}{ }_{64-2 i}
\end{aligned}
$$

The author gives also a corresponding scheme for the analysis of a function defined by 120 equally spaced ordinates. This involves the use of the machine four times.

The paper contains 6 cuts taken from drawings made by the machine. Amplitudes as small as $1 / 10 \mathrm{~mm}$. are said to be discernible. For extreme sensitivity in drawing, the tracing point is replaced by a beam of light sharply focused on photographic paper. As indicated above however, drawing curves is an activity of the machine which is of secondary importance to the business of accurate harmonic analysis, but is of much more importance to the other uses to which the machine is put. These will be taken up in a later review.

D. H. L.

1 A. A. Michelson and S. W. Stratron, "A new harmonic analyzer," Amer. Jn. Sci., v. 155 , or s. 4 , v. 5,1898 , p. $1-13$ + plate.

7[Z].-S. L. Brown and Lisle Lorenzo Wheeler (1904- ), "A mechanical method for graphical solution of polynomials," Franklin Institute, Jn., v. 231, 1941, p. 223-243.

This paper discusses the application of the harmonic synthesizer, described in the preceding review, to the problem of graphing a polynomial function of a complex variable with a view to obtaining the roots of the polynomial.

The method is one suggested in 1928 by A. J. Kempner and applied by a specially constructed machine, designed by T. C. Fry, by the Bell Telephone Laboratories and called the Isograph. ${ }^{1}$

The present machine is capable of handling equations up to the 15th degree.

Briefly the method is as follows.

Let

$$
z=r(\cos \theta+i \sin \theta)
$$


be a complex variable. If

$$
f(x)=\sum_{n=0}^{k} a_{n} x^{n}=0
$$

is the equation under discussion, the machine is set to draw the map $M$ in the $w$-plane of the circle

under the transformation

$$
|z|=r
$$

$$
w=\sum_{n=1}^{k} a_{n} r^{n}(\cos n \theta+i \sin n \theta) .
$$

The number of times this map encircles the point $w=-a_{0}$ is the number of roots within the circle $|z|=r$. By choosing different values of $r$ and inspecting the different maps, a value of $r$ is soon found whose map passes through the point $w=-a_{0}$. This value of $r$ is the absolute value of one root of $f(x)=0$. The argument of this root is the angle $\theta$ read on the machine just as the tracing point reaches the point $w=-a_{0}$.

To draw these maps the sine elements of the machine are used to drive the tracing point up and down while the cosine elements are used to drive the drawing board back and forth. The amplitude (for a fixed $r$ ) set on the $n$th crosshead is $\left|a_{n}\right| r^{n}$. If $a_{n}$ is not real, its argument is set by giving the crosshead an appropriate initial angular displacement. A dozen or more of these maps are illustrated in the paper.

A second method applicable to real roots only is given. Suppose that one wishes to find all the real roots of $f(x)=0$ that lie between $-r$ and $+r$. Substitute $x=r \cos \theta$ in

$f(x)=\sum_{n=0}^{k} a_{n} x^{n}$. Since $\cos ^{n} \theta$ is a linear combination of $\cos n \theta, \cos (n-2) \theta, \cdots$, the poly-

nomial $f(x)$ is reduced to a series of cosines which the machine can draw in one application. The real roots of $f(x)=0$ (and indeed any other features of the graph of $f(x)$ for $-r \leq x \leq r$ ) can then be read from this graph. The paper offers convincing evidence that this harmonic synthesizer is a powerful tool for studying polynomials and their roots.

D. H. L.

${ }^{1}$ Bell Laboratories Record, v. 16, 1937, R. L. Dietzold, "The isograph-a mechanical root-finder," p. 130-134; R. O. MERCNER, "The mechanism of the isograph," p. 135-140.

\section{NOTES}

10. BRIGGS AND VietA.-That Briggs in early years of the seventeenth century should have computed a "canon of sines," and of other functions, for every hundredth of a degree in the quadrant, is a notable fact, and that he was probably mainly led to this choice of argument by a passage in Vieta's Relatio Calendarii vere Gregoriani, Paris, 1600 is of special interest. The passage on p. 1 of his Trigonometria Britannica (1633) where Briggs tells us this is, in translation from the Latin, as follows: "Therefore we divide any circumference in to 360 parts which we call degrees; and each degree we divide sexagesimally into minutes and seconds, etc. But $\mathrm{I}$, induced by the authority of Vieta 'pag. 29. Calendarij Gregoriani,' and on the advice of others, divide degrees by the decimal system into 100 primary parts, and each of these into 10 parts, of which each one is divided in the same way. And these parts give a much easier, and not less certain method of calculation." With too hasty a glance at this passage I made an incorrect statement (which might have been corrected with the change of a single word) on page 33, line 12; see Corrigenda, p. 100 . Since Vieta's Relatio of 1600 is 